\title{
Spectral Sufficient Conditions on Pancyclic Graphs
}

\author{
Guidong Yu $\mathbb{D},{ }^{1,2}$ Tao Yu, ${ }^{1}$ Xiangwei Xia, ${ }^{1}$ and Huan $X u^{2}$ \\ ${ }^{1}$ School of Mathmatics and Physic, Anqing Normal University, Anqing 246133, China \\ ${ }^{2}$ Department of Public Teanching, Hefei Preschool Education College, Hefei 230013, China \\ Correspondence should be addressed to Guidong Yu; guidongy@163.com
}

Received 6 May 2021; Revised 22 June 2021; Accepted 30 June 2021; Published 15 July 2021

Academic Editor: Shaohui Wang

Copyright (c) 2021 Guidong Yu et al. This is an open access article distributed under the Creative Commons Attribution License, which permits unrestricted use, distribution, and reproduction in any medium, provided the original work is properly cited.

A pancyclic graph of order $n$ is a graph with cycles of all possible lengths from 3 to $n$. In fact, it is NP-complete that deciding whether a graph is pancyclic. Because the spectrum of graphs is convenient to be calculated, in this study, we try to use the spectral theory of graphs to study this problem and give some sufficient conditions for a graph to be pancyclic in terms of the spectral radius and the signless Laplacian spectral radius of the graph.

\section{Introduction}

Let $G=(V(G), E(G))$ be a finite simple undirected graph. Write by $n=|V(G)|$ the number of vertex and $m=|E(G)|$ the number of edges of the graph $G$, respectively. Write by $\delta(G)$ the minimum degree of $G, N_{G}\left(v_{i}\right)$ the set of neighbours of a vertex $v_{i}$ in $G$, and $d_{i}=d_{G}\left(v_{i}\right)=\left|N_{G}\left(v_{i}\right)\right|$. If $\left(d_{1}, d_{2}, \ldots, d_{n}\right)$ can be the degree sequence of a graph, $d_{1} \leq d_{2} \leq \cdots \leq d_{n}$, and then, we call it a graphic sequence. Let $G$ and $H$ be two disjoint graphs. Denote by $G+H$ the disjoint union of $G$ and $H$, which is the graph with the vertex set $V(G) \cup V(H)$ and edge set $E(G) \cup E(H)$. If $G_{1}=G_{2}=\cdots=G_{k}$, we denote $G_{1}+G_{2}+$ $\cdots+G_{k}$ by $k G_{1}$. Denote by $G \vee H$ the joint of $G$ and $H$, which is the graph obtained from disjoint union of $G$ and $H$ by adding edges joining every vertex of $G$ to every vertex of $H$. Denote by $K_{n-1}+v$ the complete graph on $n-1$ vertices together with an isolated vertex $v$ and $K_{n-1}-e$ the complete graph on $n-1$ vertices deleting the edge. Graph theory is widely used in many fields $[1,2]$. Here, we mainly study using the spectral theory of graphs to determine the cycles' properties of the graph.

The adjacency matrix of $G$ of order $n$ is the matrix $A(G)=\left[a_{i j}\right]$ of order $n$, where $a_{i j}=1$ if $v_{i}$ is adjacent to $v_{j}$ and $a_{i j}=0$ otherwise. Denote by $\mu(G)$ the spectral radius of $G$, which is the largest eigenvalue of $A(G)$. Write by $D(G)$ the degree diagonal matrix of $G$. The signless Laplacian matrix of $G$ is defined to be the matrix
$Q(G)=D(G)+A(G)$. Denote by $q(G)$ the signless Laplacian spectral radius of $G$, which is the largest eigenvalue of $Q(G)$.

A graph $G$ is Hamiltonian (respectively, traceable) if it contains a cycle (respectively, path) containing all vertices of the graph. A cycle of length $l$ is called an $l-$ cycle. A graph $G$ is pancyclic if it contains all $l-$ cycle, $3 \leq l \leq n$. Indeed, it is NPcomplete that deciding whether a given graph is Hamiltonian.

Because the spectrum of graphs is convenient to be calculated, some researchers try to use the spectrum of the graph to character the Hamiltonian of graph. In 2010, Fiedler and Nikiforov [3] first gave sufficient conditions for the given graph to be Hamiltonian (or traceable) in terms of the spectral radius of the graph. In fact, it provides an easy and efficient determination method. And then, many good conclusions have been given [2, 4-14]. But there are no spectral sufficient conditions on pancyclic graphs. In this study, we first give a new sufficient condition for a graph to be pancyclic with the edge number of the graph; then basing on the edge number sufficient condition, we give spectral radius or signless Laplacian spectral radius conditions for a graph to be pancyclic.

In this study, we can use some graphs, $G_{1} \sim G_{8}$, in the proof of the theorem, as shown in Figure 1.

$G_{1}$ is the graph obtained from $K_{2} \vee\left(2 K_{1}+K_{n-3}\right)$ by adding two pedending edges on one vertex of degree $2 . G_{2}$ is 


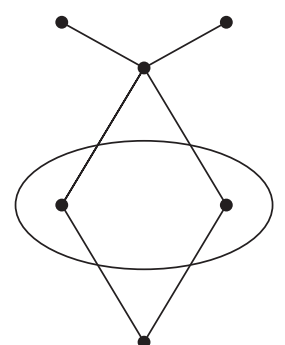

(a)

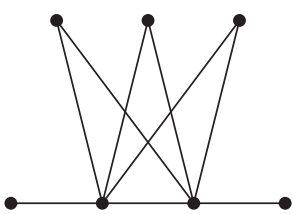

(e)

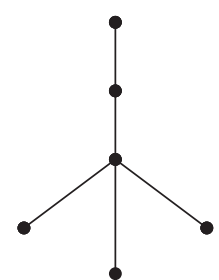

(b)

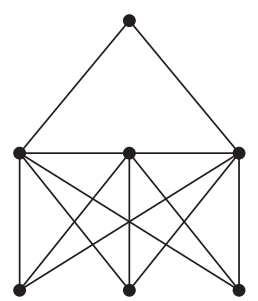

(f)

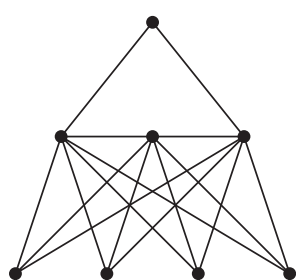

(c)

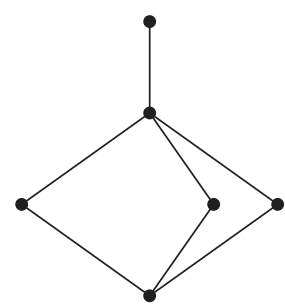

(g)

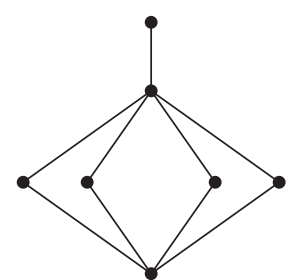

(d)

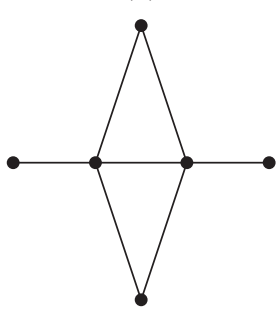

(h)

Figure 1: Some special graphs used in this study. (a) $G_{1}$. (b) $G_{2}$. (c) $G_{3}$. (d) $G_{4}$. (e) $G_{5}$. (f) $G_{6}$. (g) $G_{7}$. (h) $G_{8}$.

the graph obtained from $S_{5}$ by adding one pedending edge on one vertex of degree $1 . G_{3}$ is the graph obtained from $P_{3} \vee 4 K_{1}$ by adding all edges from two vertexes with degree 5 to $K_{1} . G_{4}$ is the graph obtained from $4 K_{1} \vee 2 K_{1}$ by adding one pedending edge on one vertex of degree $4 . G_{5}$ is the graph obtained from $K_{2} \vee 3 K_{1}$ by adding one pedending edge on two vertexes of degree 4 , respectively. $G_{6}$ is the graph obtained from $P_{3} \vee 3 K_{1}$ by adding all edges from two vertexes of degree 4 to $K_{1}$. $G_{7}$ is the graph obtained from $3 K_{1} \vee 2 K_{1}$ by adding one pedending edge on one vertex of degree $3 . G_{8}$ is the graph obtained from $K_{2} \vee 2 K_{1}$ by adding one pedending edge on two vertexes of degree 3 , respectively.

\section{Preliminary}

Given a graph $G$ with $n$ vertex, a vector $X \in R^{n}$ is called to be defined on $G$, if there is a 1-1 map $\varphi$ from $V(G)$ to the entries of $X$, simply written $X_{u}=\varphi(u)$.

If $X$ is an eigenvector of $A(G)$ (or $Q(G)$ ), then we call that $X$ is defined on $G$, and $X_{u}$ is the entry of $X$ which is corresponded to the vertex $u$. In fact, when $\lambda$ is an eigenvalue of $G$ which is corresponded to the eigenvector $X$,

$$
\lambda X_{v}=\sum_{u \in N_{G}(v)} X_{u}, \quad \text { for } v \in V(G) .
$$

We call equation (1) is eigen equation of $G$. When $q$ is a signless Laplacian eigenvalue of $G$ which is corresponded to the eigenvector $X$, we have

$$
\left[q-d_{G}(v)\right] X_{v}=\sum_{u \in N_{G}(v)} X_{u}, \quad \text { for } v \in V(G) .
$$
of $G$.

We call equation (2) is signless Laplacian eigen equation

Lemma 1 (see [15]). Let $G$ be a graph with $|V(G)|=n$, which has degree sequence $d_{1} \leq \cdots \leq d_{n}$. If for all positive integers $k$, such that $d_{k} \leq k<n / 2$ and $d_{n-k} \geq n-k$, then $G$ is a pancyclic graph or bipartite graph.

Lemma 2 (see [16]). Let $G$ be a connected graph with $|V(G)|=n$. We have

$$
\mu(G) \leq \sqrt{2|E(G)|-|V(G)|+1}
$$

and the equality holds if and only if $G=K_{n}$ or $G=K_{1, n-1}$.

Lemma 3 (see [2]). Let $G$ be a graph with $|V(G)|=n$. Then,

$$
q(G) \leq \frac{2|E(G)|}{|V(G)|-1}+|V(G)|-2 .
$$

If $G$ is connected, the equality holds if and only if $G=$ $K_{1, n-1}$ or $G=K_{n}$. Otherwise, the equality holds if and only if $G=K_{n-1}+v$.

\section{Main Results}

Theorem 1. Let $G$ be a simple connected graph, where $|V(G)|=n(\geq 5),|E(G)|=m, \delta(G) \geq 2$. If

$$
m \geq\left(\begin{array}{c}
n-2 \\
2
\end{array}\right)+3 \text {, }
$$

then $G$ is a pancyclic graph, unless $G$ is a bipartite graph or 


$$
\begin{aligned}
G \in \mathbb{N P}_{1} & =\left\{\left\{K_{3} \vee\left(K_{3}+3 K_{1}\right), K_{4} \vee\left(K_{2}+4 K_{1}\right), K_{2} \vee\left(K_{n-4}+2 K_{1}\right), 2 K_{1} \vee\left(K_{n-4}+2 K_{1}\right), K_{2} \vee\left(\left(K_{n-4}-e\right)+2 K_{1}\right),\right.\right. \\
& K_{1} \vee g_{1}, K_{1} \vee\left(K_{2}+K_{n-3}\right), K_{5} \vee 6 K_{1}, K_{3} \vee K_{2,6}, K_{4} \vee\left(K_{1}+K_{1,5}\right), K_{3} \vee\left(K_{2}+3 K_{1}\right), K_{1} \vee\left(2 K_{1} \vee\left(K_{2}+3 K_{1}\right)\right), \\
& K_{2} \vee\left(K_{1}+K_{1} \vee\left(K_{2}+2 K_{1}\right)\right), K_{2} \vee G_{2}, K_{3} \vee 5 K_{1}, K_{4} \vee 5 K_{1}, K_{3} \vee\left(K_{1}+K_{1,4}\right),\left(K_{2} \vee 2 K_{1}\right) \vee 5 K_{1}, \\
& K_{3} \vee\left(2 K_{1}+K_{1,3}\right), K_{2} \vee G_{5}, K_{2} \vee\left(K_{1}+K_{2} \vee 4 K_{1}\right), K_{2} \vee G_{4}, K_{1} \vee\left(5 K_{1} \vee\left(K_{2}+K_{1}\right)\right), K_{2} \vee G_{3}, \\
& 5 K_{1} \vee C_{4}, K_{3} \vee 4 K_{1}, K_{1,2} \vee 4 K_{1}, K_{2} \vee\left(K_{1}+K_{1,3}\right), G_{6}, 4 K_{1} \vee\left(K_{1}+K_{2}\right), K_{2} \vee\left(2 K_{2}+K_{1}\right), \\
& \left.K_{2} \vee\left(2 K_{1}+K_{1,2}\right), K_{1} \vee G_{7}, K_{1} \vee G_{8}\right\}
\end{aligned}
$$

Proof. Let $G$ satisfy the condition of theorem, but it is neither a pancyclic graph nor a bipartite graph. By Lemma 1 and the condition of theorem, there exists an positive integer $k$, such that $d_{k} \leq k<n / 2$ and $d_{n-k} \leq n-k-1$. Then,

$$
\begin{aligned}
2 m & =\sum_{i=1}^{k} d_{i}+\sum_{i=k+1}^{n-k} d_{i}+\sum_{i=n-k+1}^{n} d_{i} \\
& \leq k^{2}+(n-2 k)(n-k-1)+k(n-1) \\
& =n^{2}-n+3 k^{2}+(1-2 n) k \\
& =2\left(\begin{array}{c}
n-2 \\
2
\end{array}\right)+8-(k-2)(2 n-3 k-7) .
\end{aligned}
$$

Thus,

$$
m \leq\left(\begin{array}{c}
n-2 \\
2
\end{array}\right)+3-\frac{1}{2}((k-2)(2 n-3 k-7)-2) .
$$

Because $\left(\begin{array}{c}n-2 \\ 2\end{array}\right)+3 \leq m \leq\left(\begin{array}{c}n-2 \\ 2\end{array}\right)+3-1 / 2((k-2)$ $(2 n-3 k-7)-2), \quad(k-2)(2 n-3 k-7)-2 \leq 0, \quad$ i.e., $(k-2)(2 n-3 k-7) \leq 2$. Next, we discuss in the following four cases.

Case 1: $(k-2)((2 n-3 k-7)=2$, i.e., $k-2=2$ and $2 n-3 k-7=1$ or $k-2=1$ and $2 n-3 k-7=2$. We can get $k=3, n=9$ or $k=4, n=10$.

In this time, $m=\left(\begin{array}{c}n-2 \\ 2\end{array}\right)+3$, and all inequalities in the above should take mark of equality.

Case 1.1: $k=3$ and $n=9$. Then, $G$ is a graph with $d_{1}=d_{2}=d_{3}=3, \quad d_{4}=d_{5}=d_{6}=5, \quad$ and $d_{7}=d_{8}=d_{9}=8$. The three vertices with degree 8 must be adjacent to every vertex, and it induces $K_{3}$. The three vertices with degree 3 are not adjacent to other vertices, and they induce $3 K_{1}$. For the rest of 3 vertices with degree 5 , in order to make sure the requirement of degree 5 , they must be adjacent to each other and induce $K_{3}$. By the above analysis, $G$ must be isomorphic to $K_{3} \vee\left(K_{3}+3 K_{1}\right)$, as given in Table 1 . Case 1.2: $k=4$ and $n=10$. Then, $G$ is a graph with $d_{1}=d_{2}=d_{3}=d_{4}=4, \quad d_{5}=d_{6}=5$, and $d_{7}=d_{8}=$ $d_{9}=d_{10}=9$. The four vertices with degree 9 must be adjacent to every vertex, and they induce $K_{4}$. The four vertices with degree 4 are not adjacent to other vertices, and they induce $4 K_{1}$. For the remaining 2 vertices of degree 5 , in order to make sure the requirement of the degree 5 , they must be adjacent to each other, and they induce $K_{2}$. By the above analysis, $G$ must be isomorphic to $K_{4} \vee\left(K_{2}+4 K_{1}\right)$, as given in Table 1.

Case 2: $(k-2)(2 n-3 k-7)=1$, i.e., $k-2=1$ and $2 n-3 k-7=1$. We cannot find $k$ and $n$ fill the bill.

Case 3: $(k-2)(2 n-3 k-7)=0$, i.e., $k-2=0$ or $2 n-3 k-7=0$.

Case 3.1: $k-2=0$, i.e., $k=2$. Thus, $d_{2} \leq 2, d_{n-2} \leq n-3$ and $(n-2)(n-3)+6 \leq \sum_{i=1}^{n} d_{i} \leq(n-2)(n-3)+8$. Because $\sum_{i=1}^{n} d_{i}$ is even number, thus $\sum_{i=1}^{n} d_{i}=(n-$ 2) $(n-3)+8$ or $\sum_{i=1}^{n} d_{i}=(n-2)(n-3)+6$. We can get their graphic sequence and corresponding graphs as given in Table 2 .

Case 3.2: $2 n-3 k-7=0$. Then, $n \leq 13$ because $k<n / 2$, and $n=11$ and $k=5$ or $n=8$ and $k=3$.

Case 4: $(k-2)(2 n-3 k-7)<0$, i.e., $k \geq 3$ and $2 n-3 k-7<0$. In this case, we have $6 \leq 2 k<n \leq 13$.

Case 4.1: $n=13$. We have $k \leq 6,2 n-3 k-7>0$. Case 4.2: $n=12$. We have $k \leq 5,2 n-3 k-7>0$. Case 4.3: $n=11$. We have $k \leq 5,2 n-3 k-7 \geq 0$. Case 4.4: $n=10$. We have $k \leq 4,2 n-3 k-7>0$. Case 4.5: $n=8$. We have $k \leq 3,2 n-3 k-7=0$. The above five cases all contradict to $2 n-3 k-7<0$.

Case 4.6: $n=9$. Then, $k \leq 4$. When $k=3$, then $2 n-3 k-7=2>0, \quad$ in contradiction with $2 n-3 k-7<0$.

When $k=4$, then $2 n-3 k-7=-1<0$; thus, $d_{4} \leq 4$, $d_{5} \leq 4$, and we have $48 \leq \sum_{i=1}^{9} d_{i} \leq 52$ by (5) and (8). Because $\sum_{i=1}^{9} d_{i}$ is an even number, so $\sum_{i=1}^{9} d_{i}=52$ or $\sum_{i=1}^{9} d_{i}=50$ or $\sum_{i=1}^{9} d_{i}=48$. We can get their graphic sequence and corresponding graphs as given in Table 5.

Case 4.7: $n=7$. Then, $k \leq 3$, and because $k \geq 3$, i.e., $k=3$. Thus, $d_{3} \leq 3, d_{4} \leq 3$, and $26 \leq \sum_{i=1}^{7} d_{i} \leq 30$ by (5) and (8). Because $\sum_{i=1}^{7} d_{i}$ is an even number, so $\sum_{i=1}^{7} d_{i}=30$ or $\sum_{i=1}^{7} d_{i}=28$ or $\sum_{i=1}^{7} d_{i}=26$. We can get their graphic sequence and corresponding graphs as given in Table 6 .

In Tables 1-6, we can find the following:

(i) The corresponding graphs of the graphic sequence $(5,5,5,5,5,5,8,10,10,10,10)$ contain cycles of every length $l, 3 \leq l \leq 11$ 
TABle 1: The graphic sequence of $G$ and its corresponding graph.

\begin{tabular}{lcr}
\hline & Graphic sequence & Nonpancyclic \\
\hline$n=9$ & $(3,3,3,5,5,5,8,8,8)$ & $G \cong K_{3} \vee\left(K_{3}+3 K_{1}\right)$ \\
$k=3$ & & $G \cong K_{4} \vee\left(K_{2}+4 K_{1}\right)$ \\
$2 m=48$ & $(4,4,4,4,5,5,9,9,9,9)$ & \\
\hline$n=10$ & & \\
\hline$m=62$ & & \\
\hline
\end{tabular}

TABLE 2: The graphic sequence of $G$ and its corresponding graph.

\begin{tabular}{lcc}
\hline & Graphic sequence & Nonpancyclic \\
\hline$|V(G)|=n$ & $(2,2, n-3, n-3, n-3, \ldots, n-3, n-1, n-1)$ & $G \cong K_{2} \vee\left(K_{n-4}+2 K_{1}\right)$ \\
$k=2$ & $(2,2, n-3, n-3, n-3, \ldots, n-3, n-2, n-2)$ & \\
$2 m=(n-2)(n-3)+8$ & $(2,2, n-4, n-4, n-3, \ldots, n-3, n-1, n-1)$ & $G \cong 2 K_{1} \vee\left(K_{n-4}+2 K_{1}\right)$ \\
$|V(G)|=n$ & $(2,2, n-4, n-3, n-3, \ldots, n-3, n-2, n-1)$ & $G \cong K_{2} \vee\left(\left(K_{n-4}-e\right)+2 K_{1}\right)$ \\
$k=2$ & $(2,2, n-3, n-3, n-3, \ldots, n-3, n-3, n-1)$ & $G \cong K_{1} \vee G_{1}$ \\
$2 m=(n-2)(n-3)+8$ & & $G \cong K_{1} \vee\left(K_{2}+K_{n-3}\right)$ \\
\hline
\end{tabular}

TABle 3: The graphic sequence of $G$ and its corresponding graph.

\begin{tabular}{lcc}
\hline & Graphic sequence & Nonpancyclic \\
\hline$n=11$ & & \\
$k=5$ & $(5,5,5,5,5,5,10,10,10,10,10)$ & $G \cong K_{5} \vee 6 K_{1}$ \\
$2 m=80$ & & \\
\hline$n=11$ & $(5,5,5,5,5,5,9,9,10,10,10)$ & $G \cong K_{3} \vee K_{2,6}$ \\
$k=5$ & $(5,5,5,5,5,5,8,10,10,10,10)$ & $\begin{array}{c}\text { Every graph is } \\
\text { pancyclic }\end{array}$ \\
$2 m=78$ & $(4,5,5,5,5,5,9,10,10,10,10)$ & $G \cong K_{4} \vee\left(K_{1}+K_{1,5}\right)$ \\
\hline
\end{tabular}

TABLE 4: The graphic sequence of $G$ and its corresponding graph.

\begin{tabular}{lcc}
\hline & Graphic sequence & Nonpancyclic \\
\hline$n=8$ & & \\
$k=3$ & $(3,3,3,4,4,7,7,7)$ & $G \cong K_{3} \vee\left(K_{2}+3 K_{1}\right)$ \\
$2 m=38$ & & \\
\hline$n=8$ & $(3,3,3,4,4,6,6,7)$ & $G \cong K_{1} \vee\left(2 K_{1} \vee\left(K_{2}+3 K_{1}\right)\right)$ \\
& $(2,3,3,4,4,6,7,7)$ & $G \cong K_{2} \vee\left(K_{1}+K_{1} \vee\left(K_{2}+2 K_{1}\right)\right)$ \\
$k=3$ & $(3,3,3,3,4,6,7,7)$ & $G \cong K_{2} \vee G_{2}$ \\
$2 m=36$ & $(3,3,3,4,4,5,7,7)$ & Every graph is pancyclic \\
\hline
\end{tabular}

(ii) The corresponding graphs of the graphic sequence $(4,4,4,4,4,4,8,8,8)$,

$(3,4,4,4,4,5,8,8,8)$, $(4,4,4,4,4,6,6,8,8), \quad(4,4,4,4,4,5,7,8,8)$, and $(4,4,4,4,4,6,8,8,8)$ contain cycles of every length $l, 3 \leq l \leq 9$, respectively

(iii) The corresponding graphs of the graphic sequence $(3,3,3,4,4,5,7,7)$ contain cycles of every length $l$, $3 \leq l \leq 8$

(iv) The corresponding graphs of the graphic sequence $(3,3,3,3,4,4,6), \quad(3,3,3,3,3,5,6), \quad$ and
$(3,3,3,3,4,6,6)$ contain cycles of every length $l$,
TABLE 5: The graphic sequence of $G$ and its corresponding graph.

\begin{tabular}{lcc}
\hline & Graphic sequence & Nonpancyclic \\
\hline$n=9$ & & \\
$k=4$ & $(4,4,4,4,4,8,8,8,8)$ & $G \cong K_{4} \vee 5 K_{1}$ \\
$2 m=52$ & & \\
\hline$n=9$ & $(3,4,4,4,4,7,8,8,8)$ & $G \cong K_{3} \vee\left(K_{1}+K_{1,4}\right)$ \\
$k=4$ & $(4,4,4,4,4,6,8,8,8)$ & Every graph is pancyclic \\
$2 m=50$ & $(4,4,4,4,4,7,7,8,8)$ & $G \cong\left(K_{2} \vee 2 K_{1}\right) \vee 5 K_{1}$ \\
\hline & $(4,4,4,4,4,4,8,8,8)$ & Every graph is pancyclic \\
& $(3,4,4,4,4,5,8,8,8)$ & Every graph is pancyclic \\
$n=9$ & $(3,3,4,4,4,6,8,8,8)$ & $G \cong K_{3} \vee\left(2 K_{1}+K_{1,3}\right)$ \\
& $(4,4,4,4,4,6,6,8,8)$ & Every graph is pancyclic \\
& $(3,3,4,4,4,7,7,8,8)$ & $G \cong K_{2} \vee G_{5}$ \\
& $(2,4,4,4,4,7,7,8,8)$ & $G \cong K_{2} \vee\left(K_{1}+K_{2} \vee 4 K_{1}\right)$ \\
$k=4$ & $(3,4,4,4,4,6,7,8,8)$ & $G \cong K_{2} \vee G_{4}$ \\
& $(4,4,4,4,4,5,7,8,8)$ & Every graph is pancyclic \\
& $(4,4,4,4,4,6,7,7,8)$ & $G \cong K_{1} \vee\left(5 K_{1} \vee\left(K_{2}+K_{1}\right)\right)$ \\
$2 m=48$ & $(3,4,4,4,4,7,7,7,8)$ & $G \cong K_{1} \vee G_{3}$ \\
& $(4,4,4,4,4,7,7,7,7)$ & $G \cong 5 K_{1} \vee C_{4}$ \\
\hline
\end{tabular}

$3 \leq l \leq 7$, respectively. Namely, they are the pancyclic graph, a contradiction. The other graphs in Tables $1 \sim 6$ are neither pancyclic nor bipartite, and $K_{3} \vee\left(K_{3}+3 K_{1}\right)$ and $K_{4} \vee\left(K_{2}+4 K_{1}\right)$ are neither pancyclic nor bipartite.

The proof is complete.

Theorem 2. Let $G$ be a simple connected graph, where $|V(G)|=n(\geq 5),|E(G)|=m, \delta(G) \geq 2$. If

$$
\mu(G) \geq \sqrt{n^{2}-6 n+13},
$$

then $G$ is a pancyclic graph, unless $G$ is a bipartite graph or $G \in\left\{K_{3} \vee 4 K_{1}, G_{6}, K_{2} \vee 3 K_{1}\right\}$. 
TABLE 6: The graphic sequence of $G$ and its corresponding graph.

\begin{tabular}{lrr}
\hline & Graphic sequence & Nonpancyclic \\
\hline $\begin{array}{l}n=7 \\
k=3\end{array}$ & $(3,3,3,3,6,6,6)$ & $G \cong K_{3} \vee 4 K_{1}$ \\
$2 m=30$ & & $G \cong K_{1,2} \vee 4 K_{1}$ \\
\hline$n=7$ & $(3,3,3,3,5,5,6)$ & Every graph is pancyclic \\
$k=3$ & $(3,3,3,3,4,6,6)$ & $G \cong K_{2} \vee\left(K_{1}+K_{1,3}\right)$ \\
$2 m=28$ & $(2,3,3,3,5,6,6)$ & $G \cong G_{6}$ \\
$n=7$ & $(2,3,3,3,5,5,5)$ & $G \cong 4 K_{1} \vee\left(K_{1}+K_{2}\right)$ \\
& $(3,3,3,3,4,5,5)$ & $G \cong K_{2} \vee\left(2 K_{2}+K_{1}\right)$ \\
$k=3$ & $(2,3,3,3,3,6,6)$ & $G \cong K_{2} \vee\left(2 K_{1}+K_{1,2}\right)$ \\
& $(2,2,3,3,4,6,6)$ & Every graph is pancyclic \\
$2 m=26$ & $(3,3,3,3,4,4,6)$ & Every graph is pancyclic \\
& $(3,3,3,3,3,5,6)$ & $G \cong K_{1} \vee G_{7}$ \\
\end{tabular}

Proof. Let $G$ satisfy the condition of theorem, but it is neither a pancyclic graph nor a bipartite graph. Because $K_{n}$ is pancyclic and $\delta\left(K_{1, n-1}\right)=1$, by Lemma 2 ,

$$
\sqrt{n^{2}-6 n+13} \leq \mu(G)<\sqrt{2 m-n+1}
$$

then,

$$
m>\left(\begin{array}{c}
n-2 \\
2
\end{array}\right)+3
$$

By Theorem 1, we get $G \in \mathbb{N P}_{1}$. Because when $G \in\left\{K_{3} \vee\left(K_{3}+3 K_{1}\right), K_{4} \vee\left(K_{2}+4 K_{1}\right)\right\}$,

$$
m=\left(\begin{array}{c}
n-2 \\
2
\end{array}\right)+3 \text {, }
$$

is a contradiction. So,

$$
\begin{aligned}
& G \in \mathbb{N P}_{2}=\left\{K_{2} \vee\left(K_{n-4}+2 K_{1}\right), 2 K_{1} \vee\left(K_{n-4}+2 K_{1}\right), K_{2} \vee\left(\left(K_{n-4}-e\right)+2 K_{1}\right), K_{1} \vee G_{1}, K_{1} \vee\left(K_{2}+K_{n-3}\right), K_{5} \vee 6 K_{1},\right. \\
& K_{3} \vee K_{2,6}, K_{4} \vee\left(K_{1}+K_{1,5}\right), K_{3} \vee\left(K_{2}+3 K_{1}\right), K_{1} \vee\left(2 K_{1} \vee\left(K_{2}+3 K_{1}\right)\right), K_{2} \vee\left(K_{1}+K_{1} \vee\left(K_{2}+2 K_{1}\right)\right), K_{2} \vee G_{2}, K_{3} \vee 5 K_{1}, \\
& K_{4} \vee 5 K_{1}, K_{3} \vee\left(K_{1}+K_{1,4}\right), \backslash\left(K_{2} \vee 2 K_{1}\right) \vee 5 K_{1}, K_{3} \vee\left(2 K_{1}+K_{1,3}\right), K_{2} \vee G_{5}, K_{2} \vee\left(K_{1}+K_{2} \vee 4 K_{1}\right), K_{2} \vee G_{4}, \\
& K_{1} \vee\left(5 K_{1} \vee\left(K_{2}+K_{1}\right)\right), K_{2} \vee G_{3}, 5 K_{1} \vee C_{4}, K_{3} \vee 4 K_{1}, K_{1,2} \vee 4 K_{1}, K_{2} \vee\left(K_{1}+K_{1,3}\right), \\
&\left.G_{6}, 4 K_{1} \vee\left(K_{1}+K_{2}\right), K_{2} \vee\left(2 K_{2}+K_{1}\right), K_{2} \vee\left(2 K_{1}+K_{1,2}\right), K_{1} \vee G_{7}, K_{1} \vee G_{8}\right\}
\end{aligned}
$$

(i) For $K_{2} \vee\left(K_{n-4}+2 K_{1}\right)$, by theorem condition, we have $n \geq 5$. It is easy to check by WolframAlpha (http://www.wolframalpha.com) that $\mu\left(K_{2} \vee\right.$ $\left.\left(K_{n-4}+2 K_{1}\right)\right)$ is the largest root of $f(x)=x^{3}+(4-n) x^{2}-(n+1) x+4 n-20, \quad$ and $f^{\prime}(x)=3 x^{2}+(8-2 n) x-n-1$. The roots of $f^{\prime}(x)=0$ are $x_{1}=1 / 3\left(-\sqrt{n^{2}-5 n+19}+n-4\right)$ and $x_{2}=1 / 3\left(\sqrt{n^{2}-5 n+19}+n-4\right)$. Then by analyzing, when $x>x_{2}$, we have $f^{\prime}(x)>0$, so $f(x)$ is monotonically increasing. We can get when $n=5$, $f\left(\sqrt{n^{2}-6 n+13}\right)<0, \quad$ and when $n \geq 6$, $f\left(\sqrt{n^{2}-6 n+13}\right)>0$. Because $\mu(G) \geq \sqrt{n^{2}-6 n+13}$, then $n=5, G \cong K_{2} \vee 3 K_{1}$.

(ii) For $2 K_{1} \vee\left(K_{n-4}+2 K_{1}\right)$, by theorem condition, we have $n \geq 5$. Using the same method, $\mu\left(2 K_{1} \vee\left(K_{n-4}+2 K_{1}\right)\right)$ is the largest root of $f(x)=x^{3}+(5-n) x^{2}+(4-2 n) x+4 n-20, \quad$ and $f^{\prime}(x)=3 x^{2}+(10-2 n) x+4-2 n$. The roots of $f^{\prime}(x)=0$ are $x_{1}=1 / 3\left(-\sqrt{n^{2}-4 n+13}+n-5\right)$ and $x_{2}=1 / 3\left(\sqrt{n^{2}-4 n+13}+n-5\right)$. Then by analyzing, when $x>x_{2}$, we have $f^{\prime}(x)>0$, so $f(x)$ is monotonically increasing. Because when $n \geq 5$, we have $f\left(\sqrt{n^{2}-6 n+13}\right)>0$; this implies $\mu\left(2 K_{1} \vee\left(K_{n-4}+2 K_{1}\right)\right)<\sqrt{n^{2}-6 n+13}, \quad$ a contradiction.

(iii) For $K_{2} \vee\left(\left(K_{n-4}-e\right)+2 K_{1}\right)$, we can get $n \geq 6$ from the graph structure. Using the same method, we get $\mu\left(K_{2} \vee\left(\left(K_{n-4}-e\right)+2 K_{1}\right)\right)$ is the largest root of $f$ $(x)=x^{4}+(-n+6) x^{3}+(9-3 n) x^{2}+(2 n-20) x+$ $8 n-48$. Then by analyzing, $f\left(\sqrt{n^{2}-6 n+13}\right)>0$, 
and this implies $\mu\left(K_{2} \vee\left(\left(K_{n-4}-e\right)+2 K_{1}\right)\right)$ $<\sqrt{n^{2}-6 n+13}$, a contradiction.

(iv) For $K_{1} \vee G_{1}$, we can get $n \geq 6$ from the graph structure. Using the same method, we get $\mu\left(K_{1} \vee G_{1}\right)$ is the largest root of $f(x)=x^{5}+(6-$ n) $x^{4}+(9-$

$3 n) x^{3}+(2 n-20) x^{2}+(8 n-42) x+2 n-8$. By analyzing, when $n \geq 6$, then $f\left(\sqrt{n^{2}-6 n+13}\right)>0$, so $\mu\left(K_{1} \vee G_{1}\right)<\sqrt{n^{2}-6 n+13}$, a contradiction.

(v) For $K_{1} \vee\left(K_{2}+K_{n-3}\right)$, by theorem condition, we have $n \geq 5$. Using the same method, we have $\mu\left(K_{1} \vee\left(K_{2}+K_{n-3}\right)\right)$ is the largest root of $f(x)=x^{3}+(3-n) x^{2}-3 x+3 n-11$, and $f^{\prime}(x)=$ $3 x^{2}+(6-2 n) x-3$. The roots of $f^{\prime}(x)=0$ are $x_{1}=1 / 3\left(-\sqrt{n^{2}-6 n+18}+n-3\right), \quad$ and $x_{2}=1 / 3$ $\left(\sqrt{n^{2}-6 n+18}+n-3\right)$. Then by analyzing, when $x>x_{2}$, we have $f^{\prime}(x)>0$, so $f(x)$ is monotonically increasing. Because when $n \geq 5$, we have $f\left(\sqrt{n^{2}-6 n+13}\right)>0$; this implies $\mu\left(K_{1} \vee\left(K_{2}+\right.\right.$ $\left.\left.K_{n-3}\right)\right)<\sqrt{n^{2}-6 n+13}$, a contradiction.

(vi) For $\quad G=\left(K_{2} \vee 2 K_{1}\right) \vee 5 K_{1}$,

let $X=\left(X_{1}, X_{2}, \ldots, X_{9}\right)^{T}$ be the eigenvector corresponding to $\mu(G)$, where $X_{i}(1 \leq i \leq 5)$ correspond to the vertex of degree $4, X_{i}(6 \leq i \leq 7)$ correspond to the vertex of degree 7 , and $X_{i}(8 \leq i \leq 9)$ correspond to the vertex of degree 8 . Then, by eigen equation (1), we have

$$
\left\{\begin{array}{l}
X_{1}=X_{2}=\cdots=X_{5}, X_{6}=X_{7}, X_{8}=X_{9}, \\
\mu(G) X_{1}=2 X_{6}+2 X_{8}, \\
\mu(G) X_{6}=5 X_{1}+2 X_{8}, \\
\mu(G) X_{8}=5 X_{1}+2 X_{6}+X_{8} .
\end{array}\right.
$$

Transform above into a matrix equation $\left(A^{\prime}(G)-\mu(G) I\right) X^{\prime}=0$, where $X^{\prime}=\left(X_{1}, X_{6}, X_{8}\right)^{T}$ and

$$
A^{\prime}(G)=\left[\begin{array}{lll}
0 & 2 & 2 \\
5 & 0 & 2 \\
5 & 2 & 1
\end{array}\right] \text {. }
$$

Let $f(x):=\operatorname{det}\left(x I-A^{\prime}(G)\right)$; then, $f(x)=x^{3}-x^{2}-$ $24 x-30$, and $\mu(G)$ is the largest root of $f(x)=0$. Through calculation, $\mu(G)=5.9150<\sqrt{9^{2}-6 \times 9+13}=6.3246$, a contradiction.

Using the same method, we get the spectral radius of the other graphs in $\mathbb{N P}_{2}$, as given inTable 7 .

From Table 7, all graphs in $\mathbb{N P}_{2}$ satisfy $\mu(G)<\sqrt{n^{2}-6 n+13}$, except $G \in\left\{K_{3} \vee 4 K_{1}, G_{6}, K_{2} \vee 3 K_{1}\right\}$, a contradiction.

The proof is complete.

Theorem 3. Let $G$ be a simple connected graph, where $|V(G)|=n(\geq 5),|E(G)|=m$, and $\delta(G) \geq 2$. If

$$
q(G) \geq \frac{6}{n-1}+2 n-6
$$

then $G$ is a pancyclic graph, unless $G$ is a bipartite graph or $G \in\left\{K_{3} \vee\left(K_{1,4}+K_{1}\right), K_{4} \vee 5 K_{1}, K_{3} \vee 5 K_{1}, K_{3} \vee 4 K_{1}, K_{3} \vee\right.$ $\left(K_{2}+3 K_{1}\right), \quad K_{2} \vee\left(2 K_{1}+K_{1,2}\right), \quad K_{2} \vee\left(K_{1}+K_{1,3}\right)$, $\left.K_{2} \vee\left(K_{n-4}+2 K_{1}\right)(5 \leq n \leq 11), K_{1} \vee 2 K_{2}\right\}$.

Proof. Let $G$ satisfy the condition of theorem, but it is neither a pancyclic graph nor a bipartite graph. Because $K_{n}$ is pancyclic and $\delta\left(K_{1, n-1}\right)=1, \delta\left(K_{n-1}+v\right)=0$. By Lemma 3,

$$
\frac{6}{n-1}+2 n-6 \leq q(G)<\frac{2 m}{n-1}+n-2 ;
$$

then,

$$
m>\left(\begin{array}{c}
n-2 \\
2
\end{array}\right)+3 .
$$

By Theorem 1 , we get $G \in \mathbb{N P}_{1}$. Because when $G \in\left\{K_{3} \vee\left(K_{3}+3 K_{1}\right), K_{4} \vee\left(K_{2}+4 K_{1}\right)\right\}$,

$$
m=\left(\begin{array}{c}
n-2 \\
2
\end{array}\right)+3
$$

a contradiction. So,

$$
\begin{aligned}
G \in \mathbb{N P}_{2} & =\left\{K_{2} \vee\left(K_{n-4}+2 K_{1}\right), 2 K_{1} \vee\left(K_{n-4}+2 K_{1}\right), K_{2} \vee\left(\left(K_{n-4}-e\right)+2 K_{1}\right), K_{1} \vee G_{1}, K_{1} \vee\left(K_{2}+K_{n-3}\right), K_{5} \vee 6 K_{1},\right. \\
& K_{3} \vee K_{2,6}, K_{4} \vee\left(K_{1}+K_{1,5}\right), K_{3} \vee\left(K_{2}+3 K_{1}\right), K_{1} \vee\left(2 K_{1} \vee\left(K_{2}+3 K_{1}\right)\right), K_{2} \vee\left(K_{1}+K_{1} \vee\left(K_{2}+2 K_{1}\right)\right), \\
& K_{2} \vee G_{2}, K_{3} \vee 5 K_{1}, K_{4} \vee 5 K_{1}, K_{3} \vee\left(K_{1}+K_{1,4}\right),\left(K_{2} \vee 2 K_{1}\right) \vee 5 K_{1}, K_{3} \vee\left(2 K_{1}+K_{1,3}\right), \\
& K_{2} \vee G_{5}, K_{2} \vee\left(K_{1}+K_{2} \vee 4 K_{1}\right), K_{2} \vee G_{4}, K_{1} \vee\left(5 K_{1} \vee\left(K_{2}+K_{1}\right)\right), K_{2} \vee G_{3}, 5 K_{1} \vee C_{4}, \\
& K_{3} \vee 4 K_{1}, K_{1,2} \vee 4 K_{1}, K_{2} \vee\left(K_{1}+K_{1,3}\right), G_{6}, 4 K_{1} \vee\left(K_{1}+K_{2}\right), K_{2} \vee\left(2 K_{2}+K_{1}\right), \\
& \left.K_{2} \vee\left(2 K_{1}+K_{1,2}\right), K_{1} \vee G_{7}, K_{1} \vee G_{8}\right\}
\end{aligned}
$$

(i) For $K_{2} \vee\left(K_{n-4}+2 K_{1}\right)$, by the theorem condition, we have $n \geq 5$. It is easy to check by Wolfram Alpha (http : //www.wolframalpha.com/) that $q\left(K_{2} \vee\left(K_{n-4}+2 K_{1}\right)\right)$ is the largest root of $f(x)=x^{3}+(-3 n+6) \quad x^{2}+\left(2 n^{2}-4 n-12\right) x-$ $4 n^{2}+28 n-48$. Then, $f(6 / n-1+2 n-6)=$ 
TABle 7: The spectral radius of $G$.

\begin{tabular}{|c|c|c|c|c|c|}
\hline G & $\mu(G)$ & $\sqrt{n^{2}-6 n+13}$ & G & $\mu(G)$ & $\sqrt{n^{2}-6 n+13}$ \\
\hline$K_{1,2} \vee 4 K_{1}$ & 4.2182 & 4.4721 & $K_{4} \vee 5 K_{1}$ & 6.2170 & 6.3246 \\
\hline$K_{2} \vee\left(K_{1}+K_{1,3}\right)$ & 4.3723 & 4.4721 & $K_{3} \vee 4 K_{1}$ & 4.6056 & 4.4721 \\
\hline$K_{5} \vee 6 K_{1}$ & 7.8310 & 8.2462 & $K_{3} \vee\left(K_{2}+3 K_{1}\right)$ & 5.1758 & 5.3852 \\
\hline$K_{3} \vee\left(K_{1}+K_{1,4}\right)$ & 6.0322 & 6.3246 & $K_{3} \vee K_{2,6}$ & 7.5826 & 8.2462 \\
\hline$K_{4} \vee\left(K_{1}+K_{1,5}\right)$ & 7.6779 & 8.2462 & $K_{1} \vee\left(2 K_{1} \vee\left(K_{2}+3 K_{1}\right)\right)$ & 4.8130 & 5.3852 \\
\hline$K_{2} \vee\left(K_{1}+K_{1} \vee\left(K_{2}+2 K_{1}\right)\right)$ & 4.9837 & 5.3852 & $K_{2} \vee G_{2}$ & 4.9273 & 5.3852 \\
\hline$K_{3} \vee 5 K_{1}$ & 5.0000 & 5.3852 & $K_{2} \vee G_{5}$ & 5.8367 & 6.3246 \\
\hline$K_{2} \vee\left(K_{1}+K_{2} \vee 4 K_{1}\right)$ & 5.8904 & 6.3246 & $K_{2} \vee G_{4}$ & 5.7407 & 6.3246 \\
\hline$K_{1} \vee\left(5 K_{1} \vee\left(K_{2}+K_{1}\right)\right)$ & 5.6293 & 6.3246 & $K_{1} \vee G_{3}$ & 5.7165 & 6.3246 \\
\hline $5 K_{1} \vee C_{4}$ & 5.5826 & 6.3246 & $K_{3} \vee\left(2 K_{1}+K_{1,3}\right)$ & 5.8503 & 6.3246 \\
\hline$G_{6}$ & 8.2749 & 4.4721 & $4 K_{1} \vee\left(K_{1}+K_{2}\right)$ & 3.8482 & 4.4721 \\
\hline$K_{2} \vee\left(2 K_{2}+K_{1}\right)$ & 4.0839 & 4.4721 & $K_{2} \vee\left(2 K_{1}+K_{1,2}\right)$ & 4.1377 & 4.4721 \\
\hline$K_{1} \vee G_{7}$ & 3.9964 & 4.4721 & $K_{1} \vee G_{8}$ & 4.1160 & 4.4721 \\
\hline
\end{tabular}

$4\left(n^{4}-18 n^{3}+90 n^{2}-211 n+192\right) /(n-1)^{3}$. When $5 \leq n \leq 11, f(6 / n-1+2 n-6)<0$, and when $n \geq 12$, $f(6 / n-1+2 n-6)>0$. Because $q(G) \geq 6 / n-$ $1+2 n-6$, then $5 \leq n \leq 11$, and $G=K_{2} \vee$ $\left(K_{n-4}+2 K_{1}\right)$.

(ii) For $2 K_{1} \vee\left(K_{n-4}+2 K_{1}\right)$ because $n \geq 5$. Using the same method, we have $q\left(2 K_{1} \vee\left(K_{n-4}+2 K_{1}\right)\right)$ is the largest root of $f(x)=x^{3}+(-3 n+8) x^{2}+\left(2 n^{2}-8 n\right)$ $x-4 n^{2}+36 n-80$. Then, $f(6 / n-1+2 n-6)=$ $4(n-4)\left(3 n^{3}-14 n^{2}+34 n-41\right) /(n-1)^{3}>0$. So, $q\left(2 K_{1} \vee\left(K_{n-4}+2 K_{1}\right)\right)<6 / n-1+2 n-6, \quad$ a contradiction.

(iii) For $K_{2} \vee\left(\left(K_{n-4}-e\right)+2 K_{1}\right)$ because $n \geq 6$. Using the same method, we get $q\left(K_{2} \vee\left(\left(K_{n-4}-e\right)+2 K_{1}\right)\right)$ is the largest root of $f(x)=x^{4}+(-4 n+12) x^{3}+\quad\left(5 n^{2}-28 n+28\right)$ $x^{2}+\left(-2 n^{3}+12 n^{2}+12 n-120\right) x+4 n^{3}-$ $52 n^{2}+224 n-320$. Then, $f(6 / n-1+2 n-6)>0$. So, $q\left(K_{2} \vee\left(\left(K_{n-4}-e\right)+2 K_{1}\right)\right)<6 / n-1+2 n-6$, a contradiction.

(iv) For $K_{1} \vee G_{1}$, we can get $n \geq 6$. Using the same method, we get $q\left(K_{1} \vee G_{1}\right)$ is the largest root of $f(x)=x^{5}+(-5 n+14) \quad x^{4}+\left(9 n^{2}-48 n+54\right) x^{3}+$ $\left(-7 n^{3}+50 n^{2}-78 n-42\right) x^{2}+\left(2 n^{4}-12 n^{3}-36 n^{2}+\right.$ $346 n-544) x-4 n^{4}+60 n^{3}-332 n^{2}+796 n-688$.

Then, $f(6 / n-1+2 n-6)=4\left(3 n^{8}-54 n^{7}+437 n^{6}-\right.$ $2150 n^{5}+7066 n^{4}-16131 n^{3}+25286 n^{2}-25525 n+1$ $3012) /(n-1)^{5}$. We can find that when $n \geq 6$, $f(6 / n-1+2 n-6)$ is monotone increasing because $\left.f(6 / n-1+2 n-6)\right|_{n=6}>0$; thus, $q\left(K_{1} \vee G_{1}\right)<$ $6 / n-1+2 n-6$, a contradiction.

(v) For $K_{1} \vee\left(K_{2}+K_{n-3}\right)$, by theorem condition, $n \geq 5$. Using the same method, $q\left(K_{1} \vee\left(K_{2}+K_{n-3}\right)\right)$ is the largest root of $f(x)=x^{3}+(5-3 n) x^{2}+$ $\left(2 n^{2}-n-16\right) x-6 n^{2}+34 n-44$. The roots of $f(x)=0$ are $x_{1}=1 / 2\left(-\sqrt{4 n^{2}-36 n+97}+2 n-3\right)$, $x_{2}=n-2, \quad$ and $\quad x_{3}=1 / 2\left(\sqrt{4 n^{2}-36 n+97}+\right.$
$2 n-3)<6 / n-1+2 n-6$, when $n \geq 6$. So, $q\left(K_{1} \vee\left(K_{2}+K_{n-3}\right)\right)<6 / n-1+2 n-6(n \geq 6)$; when $n=5, q\left(K_{1} \vee\left(K_{2}+K_{n-3}\right)\right)>6 / n-1+2 n-6$; and when $n \geq 6, \quad q\left(K_{1} \vee\left(K_{2}+K_{n-3}\right)\right)<6 / n-$ $1+2 n-6(n \geq 6)$. Because $q(G) \geq 6 / n-1+2 n-6$, then $n=5$, and $G=K_{1} \vee\left(K_{2}+K_{n-3}\right)=K_{1} \vee 2 K_{2}$.

(vi) For $\left(K_{2} \vee 2 K_{1}\right) \vee 5 K_{1}$, let $X=\left(X_{1}, X_{2}, \ldots, X_{9}\right)^{T}$ be the eigenvector corresponding to $q(G)$, where $X_{i}(1 \leq i \leq 5)$ correspond to the vertex of degree 4 , $X_{i}(6 \leq i \leq 7)$ correspond to the vertex of degree 7 , and $X_{i}(8 \leq i \leq 9)$ correspond to the vertex of degree 8 . Then, by signless Laplacian eigen equation (2), we have

$$
\left\{\begin{array}{l}
X_{1}=X_{2}=\cdots=X_{5}, X_{6}=X_{7}, X_{8}=X_{9}, \\
(q(G)-4) X_{1}=2 X_{6}+2 X_{8}, \\
(q(G)-7) X_{6}=5 X_{1}+2 X_{8}, \\
(q(G)-8) X_{8}=5 X_{1}+2 X_{6}+X_{8} .
\end{array}\right.
$$

Transform above into a matrix equation $\left(Q^{\prime}(G)-q(G) I\right) \widetilde{X}=0$, where $\widetilde{X}=\left(X_{1}, X_{6}, X_{8}\right)^{T}$ and

$$
Q^{\prime}(G)=\left[\begin{array}{lll}
4 & 2 & 2 \\
5 & 7 & 2 \\
5 & 2 & 9
\end{array}\right] .
$$

Let $g(x):=\operatorname{det}\left(x I-Q^{\prime}(G)\right)$, then $g(x)=x^{3}-$ $20 x^{2}+103 x-116$, and $q(G)$ is the largest root of $g(x)=0$. Through calculation, $q(G)=12.5052<6 / 9-1+2 \times 9-$ $6=12.7500$. Using the same method, we get the signless Laplacian spectral radius of the other graphs in $\mathbb{N P}_{2}$, as given in Table 8.

From Table 8, all graphs in $\mathbb{N P}_{2}$ except $G \epsilon$ $\left\{K_{3} \vee\left(K_{1,4}+K_{1}\right), K_{4} \vee 5 K_{1}, K_{3} \vee 5 K_{1}, K_{3} \vee 4 K_{1}, K_{3} \vee \quad\left(K_{2}+\right.\right.$ $\left.\left.3 K_{1}\right), \quad K_{2} \vee\left(2 K_{1}+K_{1,2}\right), \quad K_{2} \vee\left(K_{1}+K_{1,3}\right)\right\}$, other graphs satisfy $q(G)<10 / n-1+2 n-6$, a contradiction.

The proof is complete. 
TABle 8: The signless Laplacian spectral radius of $G$.

\begin{tabular}{|c|c|c|c|c|c|}
\hline$G$ & $q(G)$ & $6 / n-1+2 n-6$ & G & $q(G)$ & $6 / n-1+2 n-6$ \\
\hline$K_{1,2} \vee 4 K_{1}$ & 8.8965 & 9.0000 & $K_{4} \vee 5 K_{1}$ & 13.1789 & 12.7500 \\
\hline$K_{2} \vee\left(K_{1}+K_{1,3}\right)$ & 9.3408 & 9.0000 & $K_{3} \vee 4 K_{1}$ & 9.7720 & 9.0000 \\
\hline$K_{5} \vee 6 K_{1}$ & 16.5887 & 16.6000 & $K_{3} \vee\left(K_{2}+3 K_{1}\right)$ & 11.0959 & 10.8571 \\
\hline$K_{3} \vee\left(K_{1}+K_{1,4}\right)$ & 12.8381 & 12.7500 & $K_{3} \vee K_{2,6}$ & 16.0352 & 16.6000 \\
\hline$K_{4} \vee\left(K_{1}+K_{1,5}\right)$ & 16.3062 & 16.6000 & $K_{1} \vee\left(2 K_{1} \vee\left(K_{2}+3 K_{1}\right)\right)$ & 10.2372 & 10.8571 \\
\hline$K_{2} \vee\left(K_{1}+K_{1} \vee\left(K_{2}+2 K_{1}\right)\right)$ & 10.7116 & 10.8571 & $K_{2} \vee G_{2}$ & 10.6393 & 10.8571 \\
\hline$K_{3} \vee 5 K_{1}$ & 10.8990 & 10.8571 & $K_{2} \vee G_{5}$ & 12.4661 & 12.7500 \\
\hline$K_{2} \vee\left(K_{1}+K_{2} \vee 4 K_{1}\right)$ & 12.5303 & 12.7500 & $K_{2} \vee G_{4}$ & 12.2386 & 12.7500 \\
\hline$K_{1} \vee\left(5 K_{1} \vee\left(K_{2}+K_{1}\right)\right)$ & 11.8787 & 12.7500 & $K_{1} \vee G_{3}$ & 12.0914 & 12.7500 \\
\hline $5 K_{1} \vee C_{4}$ & 11.6235 & 12.7500 & $K_{3} \vee\left(2 K_{1}+K_{1,3}\right)$ & 12.5601 & 12.7500 \\
\hline$G_{6}$ & 8.2749 & 9.0000 & $4 K_{1} \vee\left(K_{1}+K_{2}\right)$ & 8.0000 & 9.0000 \\
\hline$K_{2} \vee\left(2 K_{2}+K_{1}\right)$ & 8.9164 & 9.0000 & $K_{2} \vee\left(2 K_{1}+K_{1,2}\right)$ & 9.0000 & 9.0000 \\
\hline$K_{1} \vee G_{7}$ & 8.5650 & 9.0000 & $K_{1} \vee G_{8}$ & 8.8405 & 9.0000 \\
\hline
\end{tabular}

\section{Conclusions}

There are many researchers who studied the spectral theory of graphs in the Hamiltonian graph, but few considered the spectral sufficient conditions on pancyclic graphs. In this study, in terms of the edge number, the spectral radius, and the signless Laplacian spectral radius of the graph, three theorems are established for a graph to be pancyclic. The proofs are also given accordingly.

\section{Data Availability}

No data were used to support this study.

\section{Disclosure}

In order to prove the originality of the manuscript, this manuscript was prepublished on Avix.

\section{Conflicts of Interest}

The authors declare that they have no conflicts of interest.

\section{Authors' Contributions}

Huan Xu contributed to the calculation and derivation of the formula in Section 3, especially for the proof of Theorem 3.

\section{Acknowledgments}

This study was supported by the Natural Science Foundation of China (11871077), NSF of Anhui Province (1808085MA04), NSF of Department of Education of Anhui Province (KJ2020A0894), and Research and Innovation Team of Hefei Preschool Education College (KCTD202001).

\section{References}

[1] J. B. Liu, S. Akram, M. Javaid et al., "Bounds of degree-based molecular descriptors for generalized F-sum graphs," Discrete Dynamics in Nature and Society, vol. 2021, Article ID 8821020, 17 pages, 2021.
[2] G. D. Yu and Y. Z. Fan, "Spectral conditions for a graph to be Hamilton-connected," Applied Mechanics and Materials, vol. 336-338, pp. 2329-2334, 2013.

[3] M. Fiedler and V. Nikiforov, "Spectral radius and Hamiltonicity of graphs," Linear Algebra and its Applications, vol. 432, no. 9, pp. 2170-2173, 2010.

[4] Y. Z. Fan and G. D. Yu, "Spectral condition for a graph to be Hamiltonian with respect to normalized laplacian," Mathematics, vol. 1-12, 2012.

[5] L. Feng, P. Zhang, H. Liu, W. Liu, M. Liu, and Y. Hu, "Spectral conditions for some graphical properties," Linear Algebra and its Applications, vol. 524, pp. 182-198, 2017.

[6] B. Li and B. Ning, "Spectral analogues of Erdős' and MoonMoser's theorems on Hamilton cycles," Linear and Multilinear Algebra, vol. 64, no. 11, pp. 2252-2269, 2016.

[7] R. Liu, W. C. Shiu, and J. Xue, "Sufficient spectral conditions on Hamiltonian and traceable graphs," Linear Algebra and iis Applications, vol. 467, pp. 254-266, 2015.

[8] M. Lu, H. Liu, and F. Tian, "Spectral radius and Hamiltonian graphs," Linear Algebra and its Applications, vol. 437, no. 7, pp. 1670-1674, 2012.

[9] V. Nikiforov, "Spectral radius and Hamiltonicity of graphs with large minimum degree," Czechoslovak Mathematical Journal, vol. 66, no. 3, pp. 925-940, 2016.

[10] G. Yu, G. Cai, M. Ye, and J. Cao, "Energy conditions for Hamiltonicity of graphs," Discrete Dynamics in Nature and Society, vol. 2014, Article ID 305164, 6 pages, 2014.

[11] B. Zhou, "Signless laplacian spectral radius and hamiltonicity," Linear Algebra and its Applications, vol. 432, no. 2-3, pp. 566-570, 2010.

[12] Q. N. Zhou, L. G. Wang, and Y. Lu, "Some sufficient conditions on Hamiltonian and traceable graphs," Advances in Mathematics, vol. 47, no. 1, pp. 31-40, 2018.

[13] B. Ning and J. Ge, "Spectral radius and Hamiltonian properties of graphs," Linear and Multilinear Algebra, vol. 63, no. 8, pp. 1520-1530, 2015.

[14] X. Feng and G. Wang, "The least signless Laplacian eigenvalue of the complements of bicyclic graphs," 2019, https://arxiv. org/abs/1907.04798.

[15] E. F. Schmeichel and S. L. Hakimi, "Pancyclic graphs and a conjecture of bondy and chvátal," Journal of Combinatorial Theory, Series B, vol. 17, no. 1, pp. 22-34, 1974.

[16] H. Yuan, "A bound on the spectral radius of graphs," Linear Algebra and its Applications, vol. 108, pp. 135-139, 1988. 